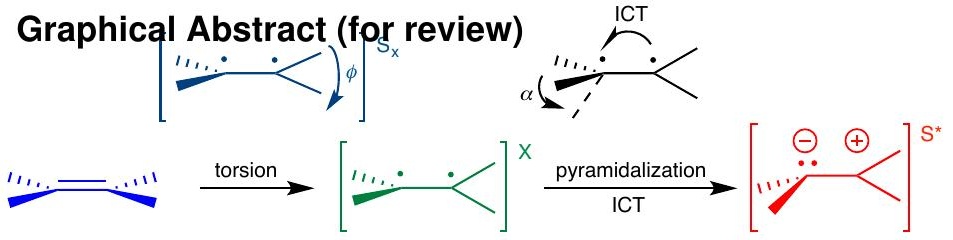




\title{
Excited State Conformational Dynamics in Carotenoids: Dark Intermediates and Excitation Energy Transfer
}

Warren F. Beck, ${ }^{*}$ Michael M. Bishop, ${ }^{\dagger}$ Jerome D. Roscioli, Soumen Ghosh, and Harry A. Frank ${ }^{\ddagger}$

Department of Chemistry, Michigan State University

578 S. Shaw Lane, East Lansing, Michigan 48824-1322 USA

†Current Address: National High Magnetic Field Laboratory, Florida State University

Tallahassee, Florida 32310 USA

‡Department of Chemistry, University of Connecticut

Storrs, Connecticut 06269-3036 USA

\begin{abstract}
A consideration of the excited state potential energy surfaces of carotenoids develops a new hypothesis for the nature of the conformational motions that follow optical preparation of the $\mathrm{S}_{2}\left(1^{1} \mathrm{~B}_{\mathrm{u}}{ }^{+}\right)$state. After an initial displacement from the Franck-Condon geometry along bond length alternation coordinates, it is suggested that carotenoids pass over a transition-state barrier leading to twisted conformations. This hypothesis leads to assignments for several dark intermediate states encountered in femtosecond spectroscopic studies. The $S_{\mathrm{x}}$ state is assigned to the structure reached upon the onset of torsional motions near the transition state barrier that divides planar and twisted structures on the $S_{2}$ state potential energy surface. The X state, detected recently in two-dimensional electronic spectra, corresponds to a twisted structure well past the barrier and approaching the $S_{2}$ state torsional minimum. Lastly, the $S^{*}$ state is assigned to a low lying $S_{1}$ state structure with intramolecular charge transfer character (ICT) and a pyramidal conformation. It follows that the bent and twisted structures of carotenoids that are found in photosynthetic light-harvesting proteins yield excited-state structures that favor the development of an ICT character and optimized energy transfer yields to (bacterio)chlorophyll acceptors.
\end{abstract}

\section{Keywords}

carotenoids; chlorophylls; energy transfer; photosynthesis; radiationless decay

\section{Abbreviations}

ICT, intramolecular charge transfer; (B)Chl, (bacterio)chlorophyll; ESA, excitedstate absorption; CARS, coherent anti-Stokes Raman scattering; SE, stimulated emission; 2DES, two-dimensional electronic spectroscopy; 


\section{Introduction}

Carotenoids are essential components in the chromoproteins of photosynthetic organisms owing to their involvement in the mechanisms of photoprotection and light harvesting [1-4]. They serve as efficient antenna chromophores in the midvisible (500-nm) spectral region where the solar spectrum is most intense. Strong, electric-dipole-allowed transitions from the carotenoid ground state, $\mathrm{S}_{0}\left(1^{1} \mathrm{Ag}_{\mathrm{g}}^{-}\right)$, to the $S_{2}\left(1^{1} \mathrm{~B}_{\mathrm{u}}{ }^{+}\right)$excited state (Figure 1$)$ are followed by downhill energy transfer to the $Q_{x}$ or $Q_{y}$ singlet states of chlorophylls (Chls) over short distances [4, 5].

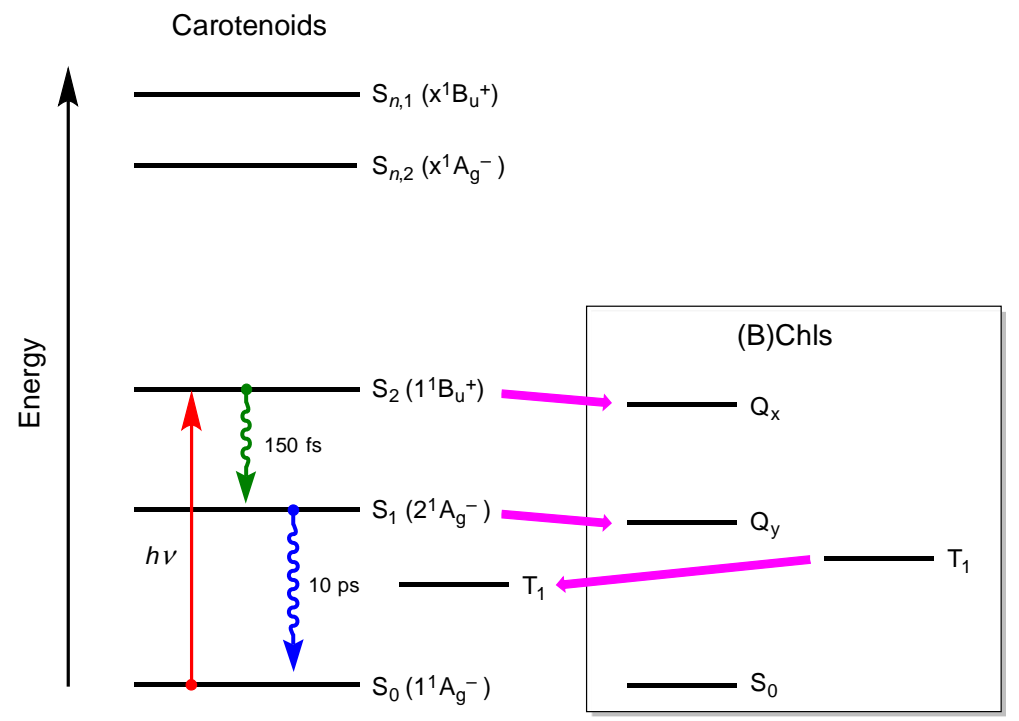

Figure 1. Energy levels and pathways for nonradiative decay in carotenoids and for excitation energy transfer from carotenoids to (B)Chls in photosynthetic lightharvesting proteins. Nonradiative decay transitions from the $S_{2}$ and $S_{1}$ states are indicated with wavy arrows. The lifetimes of the states are approximately those of $\beta$-carotene. The actual values will depend on the extent of $\pi$-electron conjugation of the carotenoid and on the nature of attached functional groups. Energy transfer pathways, forward from the carotenoid $S_{2}$ and $S_{1}$ states and reverse from the (B)Chl $\mathrm{T}_{1}$ state, are indicated with filled arrows.

The mechanisms that mediate energy transfer from the $S_{1}\left(2^{1} \mathrm{Ag}^{-}\right)$state to the $Q_{y}$ state are of considerable interest [6-8] because the $S_{1}$ state is a dark state; transitions between the $S_{1}$ and $S_{0}$ states are forbidden by symmetry. The selection rules that govern the photophysics of carotenoids apply rigorously only to the planar all-trans structures belonging to the $C_{2 h}$ point group; transitions between states of the same symmetry or pseudoparity are electric-dipole forbidden $[9,10]$. The carotenoid binding sites observed in light-harvesting and reaction-center proteins, however, contain bent and twisted structures, often in close proximity to Chls. An example of this type of interaction is shown in Figure 2 from the structure of the LH2 (B800-B850) light-harvesting protein from purple bacteria [11]. Energy transfer from the $S_{1}$ state is evidently optimized in light-harvesting proteins by 
placing the carotenoid nearly in van der Waals contact with an adjacent Chl so that the Coulomb coupling between them is as large as possible $[7,8,12,13]$. Owing to the properties of the conjugated polyene backbone, it is likely that the bent and twisted carotenoid structures further enhance the energy-transfer yield by increasing the transition dipole strength of formally dark electronic states and by promoting the development of an intramolecular charge-transfer (ICT) character.

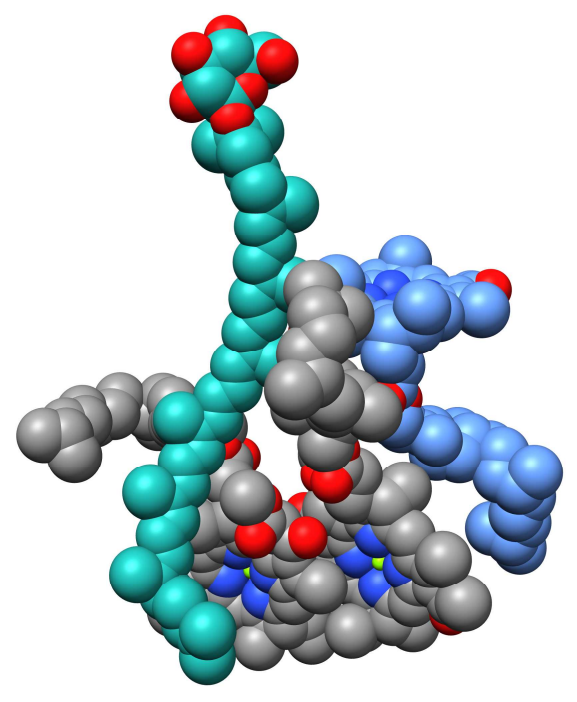

Figure 2. Interaction of rhodopin glucoside (green) with the B800 BChl monomer (blue) and the $\mathrm{B} 850 \mathrm{BChl} a$ dimer (grey) in the LH2 protein from Rhodopseudomonas acidophila (PDB entry 1NKZ) [11].

In this contribution, a hypothesis is presented that accounts for several aspects of carotenoid photophysics in terms of excited state conformational motions with respect to one or more of the conjugated $\mathrm{C}-\mathrm{C}$ and $\mathrm{C}=\mathrm{C}$ bonds. These motions naturally account for the development in the $S_{2}$ state of an ICT character within a few hundred femtoseconds of optical excitation. This picture emerges from a discussion of the excited-state potential-energy surfaces and light-driven conformational motions of shorter polyenes, trans-stilbene, and of protonated Schiff bases and cyanines. The key step in the photophysics of all these systems is an intramolecular conversion of the initial momentum in the excited state along $\mathrm{C}-\mathrm{C}$ and $\mathrm{C}=\mathrm{C}$ bond-stretching motions of the conjugated polyene into motions of the torsional coordinates, which develops the character of a biradical. This sequence of events and the dynamics that follow account for the detection via ultrafast spectroscopy of three intermediate states, $S_{x}[14], X[15]$, and $S^{*}[16]$, and suggest a possible explanation for the distinct photophysics observed in some experiments when the wavelength of the excitation pulses is tuned. Further, the distorted structures observed in light-harvesting proteins can be viewed in this picture as preparing conformations in the ground state that favor a prompt assumption of excited state ICT character. A brief discussion of the possible use of femtosecond 
nonlinear spectroscopy with heterodyne detection to address aspects of this hypothesis will also be presented.

\section{Potential Energy Surfaces and Vibrational Dynamics}

The central argument of this contribution, that carotenoids undergo ultrafast motions along torsional coordinates upon photoexcitation, is founded upon a consideration of common features of the potential energy surfaces of the singlet excited states of conjugated molecules. As noted above, however, the motions that are initially launched by the optical transition are directed from the Franck-Condon structure along the normal modes of vibration that compress the $\mathrm{C}-\mathrm{C}$ bonds and lengthen the $\mathrm{C}=\mathrm{C}$ bonds, effectively resulting in bond order reversal in the conjugated region [17-20]. This displacement initiates radiationless transitions well prior to the timescales associated with quenching of the excited state via photoisomerization.

As one important example, simulations by Levine and Martínez [21] show how the initial motions away from the Franck-Condon geometry after optical preparation of the $1^{1} \mathrm{~B}_{\mathrm{u}}$ state of trans-2-butadiene result in a strong mixing with the dark $2^{1} \mathrm{Ag}$ state. The result is an induction of transitions to and from the $1^{1} \mathrm{~B}_{\mathrm{u}}$ and $2^{1} \mathrm{Ag}$ states and an inversion of the initial energy ordering of the two states. Figure 3 describes this behavior qualitatively in terms of a crossing of diabatic potential energy curves. In $<10 \mathrm{fs}$, torsional displacements about either double bond begin and relaxation along a steep gradient in the $2^{1} \mathrm{Ag}_{\mathrm{g}}$ state occurs. A $90^{\circ}$ twisted minimum-energy structure near to a conical intersection with the ground state $\left(1^{1} \mathrm{Ag}\right)$ potential surface is assumed in $<50 \mathrm{fs}[22,23]$. Nonradiative relaxation through the conical intersection to the ground state then yields the original configuration or a photoisomerized configuration. This sequence of events is consistent with the information obtained by Trulson and Mathies [24] from resonance Raman intensity profiles, which indicate that the $1^{1} \mathrm{~B}_{\mathrm{u}}$ state undergoes a very rapid conversion to the $2^{1} \mathrm{~A}_{\mathrm{g}}$ state by moving primarily along $\mathrm{C}=\mathrm{C}$ stretching coordinates prior to the onset of out-of-plane or twisting motions. 


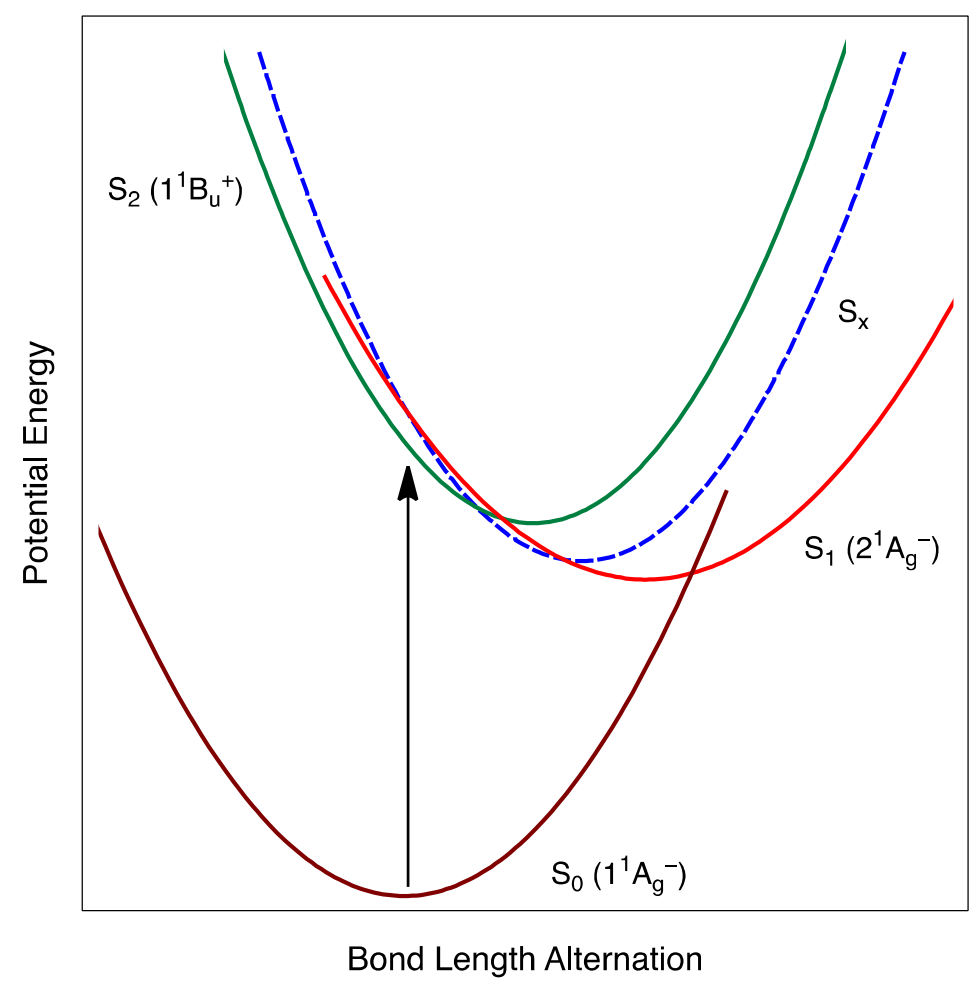

Figure 3. Diabatic energy curves for all-trans polyenes with respect to a generalized bond length alternation coordinate; after a similar diagram from Fuß et al.[20]. The dotted curve depicts the possible role in carotenoids like $\beta$-carotene of a dark intermediate state $\left(S_{x}\right)$ in the transfer of population from the bright $S_{2}\left(1^{1} B_{u^{+}}\right)$state to the dark $\mathrm{S}_{1}\left(2^{1} \mathrm{Ag}^{-}\right)$state. $\}$

For ethylene, butadiene, and the cyanines $1122-\mathrm{C}$ and $1144-\mathrm{C}$, the path from the Franck-Condon structure to a conical intersection with the ground state at the $90^{\circ}$ twisted geometry is considered barrierless [25-31]. But for trans-stilbene, an extensive body of experimental and theoretical studies indicates that a transitionstate barrier between planar and twisted conformations occurs at low torsional angles, close to the Franck-Condon geometry [32]. The descent to the twisted minimum and through the conical intersection to the $S_{0}$ state involves propagation through a potential energy region that has a doubly excited $\left(\pi^{*} \pi^{*}\right)$ character [33$35]$. In general, as the length of a conjugated polyene increases, the energy of the transition state increases because the delocalized $\pi^{*}$ character in a given C-C bond decreases. Similarly, because the $\pi^{*}$ character increases in the higher singlet states as the energy level increases, the barrier height and the modulation depth to the twisted minimum would be expected to decrease.

The analysis by Olivucci, Robb, Schlegel, and coworkers [27, 36-40] of the potential energy surfaces of models for retinal protonated Schiff bases (PSBs) and the structurally related cyanines emphasizes the crucial role of the barrier between planar and twisted conformations on the excited state dynamics. The gradients of the potential energy surface near the Franck-Condon geometry are effectively 
controlled by the conjugation length [27]. Bond length alternation and torsional forces act simultaneously in the Franck-Condon region in short polyenes, so the displacements along both coordinates are effectively coupled. With longer conjugation lengths, the Franck-Condon structure actually lies at a local minimum with respect to the torsional coordinates; the initial motions away from the FranckCondon geometry are directed only along the bond length alternation coordinates. Torsional gradients encountered near the planar minimum result in an intramolecular redistribution of the Franck-Condon structure's momentum towards twisted conformations, which promotes crossing of the activation barrier and launching of trajectories leading to the conical intersection [37]. The fluorescence of cyanines and PSBs is largely quenched by crossing to the ground state, either with isomerization or by returning to the original ground state minimum [27, 38-40].

In contrast, the model that is usually applied to the nonradiative decay of carotenoids employs a barrierless scheme like that shown in Figure 3. Only two excited states are involved, the resonant $S_{2}$ state and the dark $S_{1}$ state, and the mixing of the two states is promoted just through displacements of planar structures along bond length alternation coordinates. The contribution of torsional or out-of-plane coordinates of the conjugated polyene is usually considered minor because the $S_{1}$ state lifetime is only weakly dependent on the solvent viscosity or the temperature [19]. A consideration of results from femtosecond spectroscopies with $<50$ fs time resolution, however, suggests that it is the $S_{2}$ state that undergoes large amplitude torsional motions. Further, owing to the coupling of torsional motions with the development of ICT character, the $S_{2}$ state lifetime should be strongly affected by dynamic solvation.

\section{The $S_{x}$ and X States: Torsional Dynamics on the $S_{2}$ Potential Surface}

Several investigators have concluded that a dark electronic state, now generally labeled $S_{x}$, is populated nonradiatively from the $S_{2}$ state in $<20 \mathrm{fs}$. The reader is referred to the excellent review by Polívka and Sundström [4], which discusses the body of spectroscopic evidence for and against the existence of the $S_{x}$ state. By mediating the flow of population from the $S_{2}$ state to the $S_{1}$ state, such an intermediate state (Figure 3) would account for the deviation from the energy gap law of the rate at which the $S_{2}$ state decays to the $S_{1}$ state [41-43]. The nature of the $S_{\mathrm{x}}$ state can be inferred from the behavior of the broad excited state absorption (ESA) signal in the visible and near-IR (Figure 4), as reported by Cerullo et al. [14] in broadband femtosecond pump-probe experiments with $\beta$-carotene in cyclohexane solution. Rather than having a static profile, the ESA spectrum moves very rapidly back and forth across the visible and near-IR regions and exhibits changes in lineshape as nonradiative decay from $S_{2} \rightarrow S_{1}$ occurs.

The ESA spectrum observed by Cerullo et al. [14] in $\beta$-carotene solution initially exhibits a peak maximum at $\sim 800 \mathrm{~nm}\left(12500 \mathrm{~cm}^{-1}, 1.54 \mathrm{eV}\right)$. This spectrum $\left(\mathrm{PA}_{2}\right)$ was assigned to the $S_{2}$ state; its apparent maximum is consistent with the energy gap between the Franck-Condon $\mathrm{S}_{2}\left(1^{1} \mathrm{~B}_{u^{+}}\right)$state and an upper singlet state $S_{n, 2}$ with $g$ symmetry [44]. After a delay of only $50 \mathrm{fs}$, the peak maximum of the ESA spectrum 
moves to $\sim 975 \mathrm{~nm}\left(10250 \mathrm{~cm}^{-1}, 1.26 \mathrm{eV}\right)$, even farther into the near-IR part of the spectrum. This spectrum $\left(\mathrm{PA}_{\mathrm{x}}\right)$ is characteristic of the $\mathrm{S}_{\mathrm{x}}$ state. At $600 \mathrm{fs}$, which is near the time at which the $S_{1}$ state is maximally populated, the ESA peak $\left(\mathrm{PA}_{1}\right)$ is observed at $\sim 560 \mathrm{~nm}\left(17850 \mathrm{~cm}^{-1}, 2.20 \mathrm{eV}\right)$, and a sharper lineshape is observed. It is important to note that during this ultrafast spectral evolution associated with conversion of the $S_{2}$ state to the $S_{1}$ state, the spectral region carrying a net ESA signal is continuous (non-zero) and evidently extends beyond the blue $(500 \mathrm{~nm})$ and red $(1020 \mathrm{~nm})$ spectral limits of the available probe light in the Cerullo et al. study [14]. This behavior suggests that the ESA spectrum's spectral profile arises from a broad probability distribution with respect to torsional motions about one or more $\mathrm{C}=\mathrm{C}$ bonds.

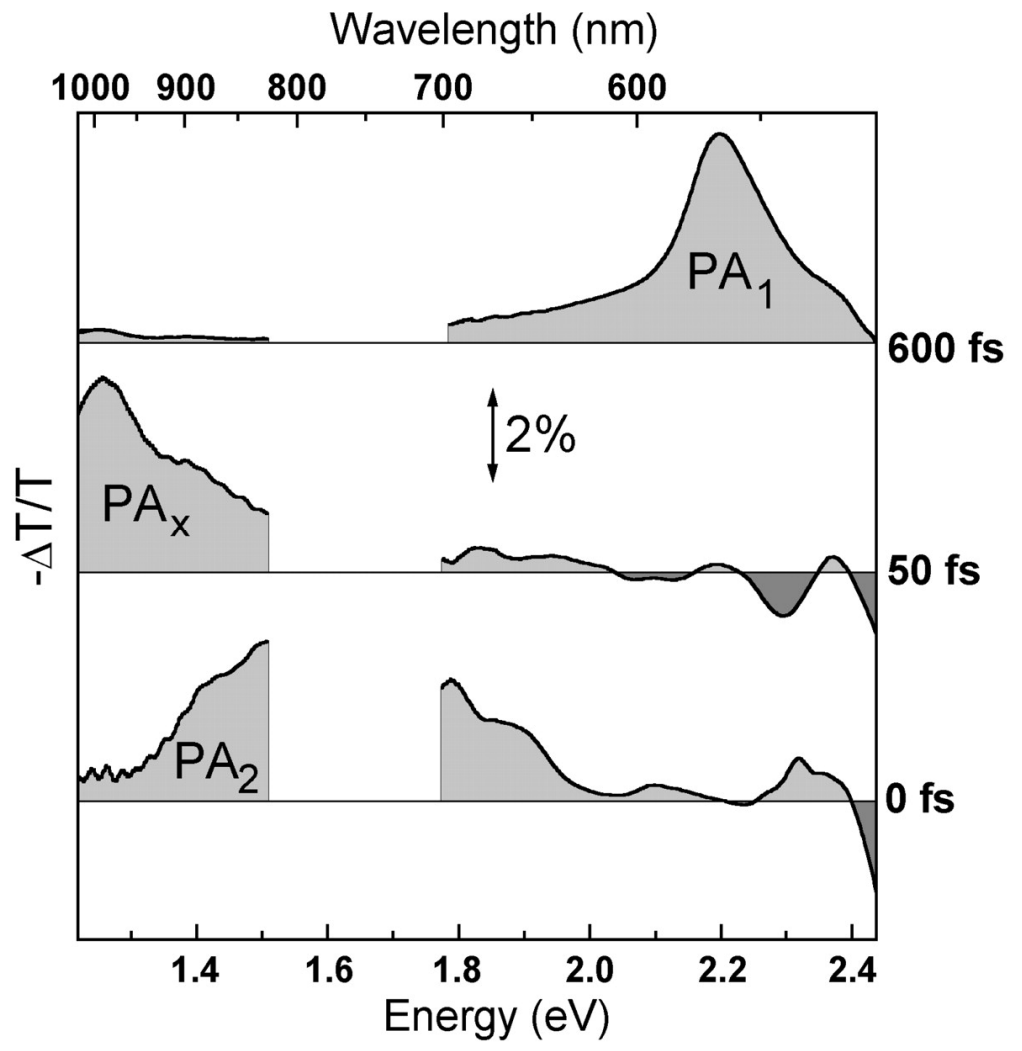

Figure 4. Time-evolution of the excited state absorption spectrum observed in cyclohexane solutions of $\beta$-carotene after optical preparation of the $S_{2}$ state. From reference [14], copyright AAAS, used with permission.

An extension of the theory of Olivucci et al. accounts for the time evolution of the ESA spectrum in carotenoids on the $<100$ fs timescale and provides an assignment for the $S_{x}$ intermediate. As in diagrams for cyanines and PSBs in the work by Sanchez-Galvez et al. [27], Figure 5 is intented to depict qualitatively the minimumenergy path on the $S_{2}$ state potential energy surface in terms of an effective reaction coordinate, with principal components along bond length alternation and torsional 
coordinates $(\phi)$. A transition-state structure $(\ddagger)$ marks the position of an activation barrier in the $S_{2}$ state that divides the planar and twisted regions of the potential energy surface. This structure is a true transition state because the initial motions from the Franck-Condon structure are only along the bond length alternation coordinates; deviation from a planar structure requires an intramolecular redistribution of momentum [36] into torsional motions, and once crossed the trajectories would be expected to lead irreversibly along torsional and out-of-plane coordinates, without recurrence to the Franck-Condon region. The potential energy curve for the $S_{n, 2}$ state, the state populated by ESA transitions from the $S_{2}$ state, is drawn above the $S_{2}$ curve; its barrier height and torsional modulation are smaller than those of the $S_{2}$ state because of the increased $\pi^{*}$ character. The $S_{0}$ potential curve exhibits local minima corresponding to the planar trans and cis structures and a barrier between them at $\phi=90^{\circ}$. The energies of the $S_{2}, S_{1}$, and $S_{0}$ states converge at this geometry, where to a first approximation a conical intersection occurs between them.

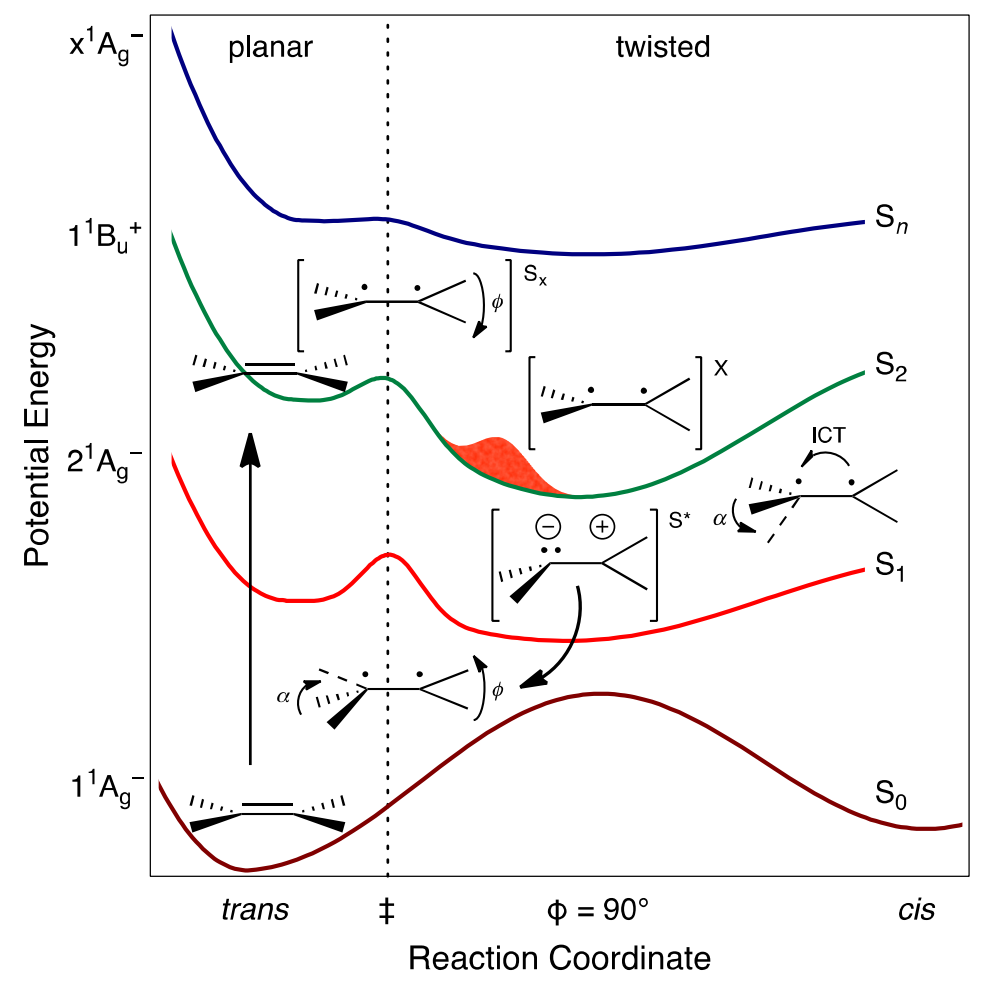

Figure 5. Proposed scheme for radiationless decay of carotenoids after optical preparation of the $S_{2}$ state; after Sanchez-Galvez et al. [27]. The states that apply to planar structures are indicated by symmetry labels. Key points along the path back to the initial planar ground state conformation are labeled with ethylenic structures, depicting the $S_{x}, X$, and $S^{*}$ dark states as twisted structures near the $S_{2}$ transition state and the $S_{2}$ twisted minimum and pyramidal structures near the $S_{1}$ state minimum, respectively. 
Applying this picture, the spectral evolution of the ESA spectrum associated with the conversion of the $S_{2}$ state to the $S_{x}$ state would be associated with the progress of a broad "wavepacket", the torsional probability distribution for one of the $\mathrm{C}=\mathrm{C}$ bonds in the conjugated backbone. The initial shift of the ESA spectral maximum from the visible to the near-IR that accompanies conversion of the $S_{2}$ state to the $S_{x}$ state is consistent with movement from the planar minimum to the the transitionstate barrier region. The position of the $\mathrm{PA}_{\mathrm{x}}$ spectral maximum at $50 \mathrm{fs}$ is well inside the $S_{0}$ to $S_{2}$ energy gap determined for planar structures. The $S_{2}$ to $S_{n, 2}$ energy gap narrows as the barrier is approached because the $S_{n, 2}$ state is displaced along the bond length alternation coordinate with respect to the $S_{2}$ state and because the height of the torsional barrier in the $S_{n, 2}$ state is lower than that of the $S_{2}$ state.

Note that an alternate interpretation of the $\mathrm{PA}_{2}$ and $\mathrm{PA}_{\mathrm{x}} \mathrm{ESA}$ spectra was discussed by Kosumi et al. [45, 46], who performed a series of femtosecond twophoton absorption experiments with nonresonant excitation (200 fs pulses) above and below the energy of the $S_{1}\left(2^{1} \mathrm{Ag}^{-}\right)$state. These experiments were designed [46] to exploit the selection rules to prepare the $S_{1}$ state directly via a two-photon allowed transition without having to populate the $S_{2}$ state first via a one-photon transition, as is usually done. With excitation to the red of $\sim 620 \mathrm{~nm}\left(16200 \mathrm{~cm}^{-1}\right.$, $2.0 \mathrm{eV}$ ), the pump-probe spectrum they observed at $0 \mathrm{fs}$ probe delay is consistent with preparation of a high vibrational energy level in the $S_{1}$ state manifold, though it is also possible that a $n^{1} \mathrm{Ag}^{-}$state in the vicinity of the $S_{2}$ state was prepared initially. Shreve and coworkers [6] had previously noted that the high polarizability of the $\mathrm{S}_{2}$ state measured in third-harmonic generation experiments is consistent with a a $g$ polarized state near to and coupled to the $S_{2}$ state.

When the excitation pulses were tuned to the blue of $\sim 620 \mathrm{~nm}$, however, Kosumi et al. observed a very broad, red-shifted pump-induced absorption spectrum spanning at least the $514-770 \mathrm{~nm}$ range (12960-19440 $\left.\mathrm{cm}^{-1}, 1.6-2.4 \mathrm{eV}\right)$. It was argued that this spectrum corresponds to the $\mathrm{PA}_{2}$ spectrum observed by Cerullo et al. with resonant excitation of the $S_{2}$ state, so it was concluded that the $\mathrm{PA}_{2}$ and $\mathrm{PA}_{\mathrm{x}}$ spectra were of "nonresonant" origin, not arising from dynamics following onephoton preparation of the $S_{2}$ state [45]. The peak maximum of the the photoinduced absorption spectrum was in the 590-685 nm (1.8-2.1 eV, $\left.14580-17010 \mathrm{~cm}^{-1}\right)$ range, however, well to the blue of the Cerullo et al. $\mathrm{PA}_{2}$ spectrum. Also, because the Kosumi et al. $[45,46]$ spectra were noted to exhibit significant contributions from coherent anti-Stokes Raman scattering (CARS), it is probable that a significant part of the signal arises from the broad non-resonant background that is always present in CARS spectra $[47,48]$. This point partly accounts for the tracking of the photoinduced absorption peak maximum with the tuning of the excitation pulse, though it is also likely that ESA to a high energy $n^{1} \mathrm{~B}_{\mathrm{u}}$ state at about $6 \mathrm{eV}(48600$ $\mathrm{cm}^{-1}$ ) above the ground state makes a contribution to the signal.

The second phase of the dynamics suggested in Figure 5 is associated with relaxation along torsional coordinates towards a twisted $S_{2}$ state minimum. This relaxation is evident in pump-continuum probe experiments by de Weerd et al. [49], who employed a target analysis [50] to isolate a broad, red-shifted stimulated 
emission (SE) band from the $S_{2}$ state of $\beta$-carotene with a 150 -fs decay time constant, which is consistent with the fluorescence lifetime of the $S_{2}$ state. The results of this work were interpreted in terms of very rapid torsional motions on the $S_{2}$ potential surface using a potential surface diagram having several of the features of Figure 5; in particular, the proposed $S_{2}$ potential energy curve has a barrier between the Franck-Condon and twisted minimum, and the phase of the torsional modulation of the $S_{0}$ potential energy curve is $\pi$ phase-shifted with respect to that of the excited-state curves, just as in ethylene but reflecting different energies for the trans and cis configurations. Thus, as torsional motions of the conjugated polyene advance past the transition state barrier, the energy gap between the $S_{2}$ state and the $S_{0}$ state narrows, so the SE would be expected to move to the red. Spectral evolution of this type is considered characteristic of torsional motions on excited state potential surfaces in conjugated molecules [25]. By $150 \mathrm{fs}$, the system would be expected to approach the twisted $S_{2}$ minimum if timescales like those in PSBs are applicable [51]. Similarly, the ESA spectrum from the $S_{2}$ state would be expected to shift to the blue after the transition state barrier is crossed; the twisted side of the potential energy surface is associated with an increase in the energy gap between $S_{2}$ and $S_{n}$; owing to its increased $\pi^{*}$ character, the barrier height and gradient in the $S_{n}$ state are smaller than in the $S_{2}$ state. As noted in the de Weerd et al. work [49], however, the regions of the spectrum carrying SE and ESA overlap extensively in pump-probe spectra, so it is difficult to monitor directly the time evolution of either spectrum on the 50 -fs timescale that would be anticipated to be associated with torsional relaxation towards the twisted minimum.

Spectral congestion can sometimes be alleviated in complex systems with overlapping spectra by applying a two-dimensional version of the technique to disperse the spectral information along two frequency axes. Accordingly, some additional crucial details of the second phase of dynamics along torsional coordinates on the $S_{2}$ potential surface (Figure 5) can be retrieved from the twodimensional electronic spectroscopy (2DES) experiments performed recently by Ostroumov et al. $[15,52]$ on carotenoids in solution and in the LH2 light-harvesting complex. The key result is the detection for the first time of an intermediate state that they call X, which exhibits off-diagonal cross-peaks in the 2DES spectra. Spectra of this type provide information on the correlation of the absorption transitions excited by a pair of excitation pulses with the absorption transitions excited by a delayed probe pulse; the cross-peaks identify the states that are connected by exchange processes, such as energy transfer [53]. The cross-peaks observed at different timescales sequentially report transfers of population from the $S_{2}$ state to the $S_{1}$ state, and, in LH2, intermolecular energy transfer to the $Q_{x}$ state of the BChl acceptor. Figure 6 shows basis-set spectra from a four-state target analysis of the 2DES spectra observed in LH2. The basis spectra describe the flow of population from the $S_{2}$ state after optical preparation either to the $S_{1}$ state or the BChl $Q_{x}$ state. 

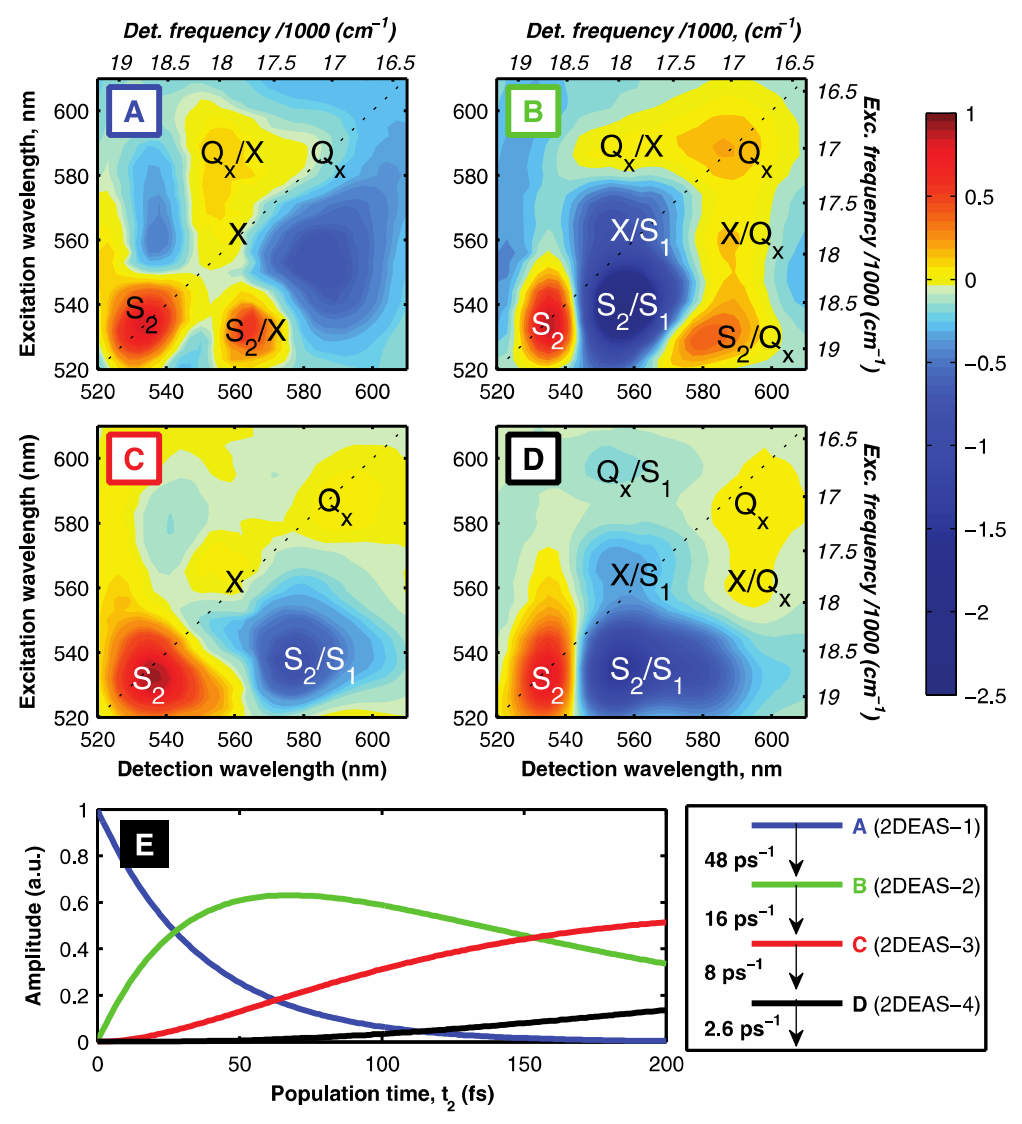

Figure 6. Basis spectra (A-D) and time evolution (E) for a four-state target analysis of the 2DES spectra from the LH2 complex of Rhodopseudomonas acidophila. From reference [15], copyright AAAS, used with permission.

The 2DES spectra observed by Ostroumov et al. [15] suggest to us that the $X$ intermediate corresponds to a twisted structure well beyond the transition state barrier shown in Figure 5 and perhaps near the minimum of the $S_{2}$ potential-energy curve. The on-diagonal peak in the 2DES spectra for photobleaching of the $\mathrm{X}$ intermediate, corresponding to a direct excitation from $S_{0}$ to $X$ at $\sim 560 \mathrm{~nm}$, indicates that the energy gap is significantly smaller than that for $S_{0}$ and $S_{2}$, as determined by the SE spectra observed by de Weerd et al. [49] farther to the blue $(500-540 \mathrm{~nm}$, depending on the solvent). The $\mathrm{X}$ absorption spectrum is consistent with a vertical optical transition to the $S_{2}$ potential surface from a ground-state structure that is conformationally displaced. The excited-state structure prepared by this transition is significantly further along the torsional coordinate than the $S_{x}$ structure. That the $\mathrm{X}$ state exhibits significant transition dipole strength to and from the ground state would be expected for a structure with a significantly lower symmetry than the planar ground state. It does not seem as obvious that the dark ${ }^{1}{ }^{1} \mathrm{~B}_{\mathrm{u}}{ }^{-}$state, the assignment suggested for $\mathrm{X}$ by Ostroumov et al., would exhibit such a red-shifted transition; it was suggested, however, that the transition dipole strength for the $\mathrm{S}_{0}$ $\left(1^{1} \mathrm{Ag}^{-}\right)$to $1^{1} \mathrm{~B}_{\mathrm{u}}^{-}$transition would be nonzero only if the Herzberg-Teller intensity 
borrowing mechanism obtained strength from the nearby $\mathrm{S}_{2}\left(1^{1} \mathrm{Bu}^{+}\right)$state through a non-symmetric vibrational distortion [15].

Further, while the diagonal region of the 2DES spectra is congested and exhibits a significant overlap of spectral regions with contributions from ESA and SE, as also noted above for 1D pump-probe spectra, a very broad off-diagonal ESA region that extends at least over the $560-610 \mathrm{~nm}$ region is partially resolved in the 2DES spectra owing to the 2D presentation. This ESA region is significantly blue shifted from the $\mathrm{PA}_{\mathrm{x}}$ spectrum discussed above, which is consistent with the expected steep expansion of the energy gap between the $S_{2}$ and $S_{n, 2}$ potential surfaces that would accompany relaxation along the torsional coordinates. The time evolution of this ESA region is significant during the $S_{2}$ to $S_{1}$ process, so its progress is informative of the nature of the associated dynamics during the four kinetic timescales detected in the target analysis described in Figure 6 [15]. The initial time constant, 20 fs (1 / 48 $\mathrm{ps}^{-1}$ ) is consistent with that detected by Cerullo et al. [14] for the formation of the $S_{\mathrm{x}}$ state; the target spectrum includes positive cross peaks linking $S_{2}$ and the $X$ intermediate. A second time constant, $62.5 \mathrm{fs}\left(1 / 16 \mathrm{ps}^{-1}\right)$, can be attributed to the formation of the $\mathrm{X}$ intermediate proper; it is probably associated with evolution further along the torsional coordinate past the barrier because the ESA region sharpens and deepens as it shifts even further to the blue, obscuring the $S_{2} / X$ cross peak observed earlier. This spectrum notably includes ESA cross peaks that connect the $S_{2}$ and $X$ states with the $Q_{x} B C h l$ acceptor state. The third and fourth time constants, $125 \mathrm{fs}\left(1 / 8 \mathrm{ps}^{-1}\right)$ and $4.62 \mathrm{ps}\left(1 / 2.6 \mathrm{ps}^{-1}\right)$, are likely to correspond to the decay of the $\mathrm{X}$ state to the $\mathrm{S}_{1}$ state and to the ground-state recovery time due to quenching by $\mathrm{BChl}_{\mathrm{X}}$ state, respectively. Note that the former timescale is comparable to the apparent fluorescence lifetime of the $S_{2}$ state of $\beta$-carotene obtained in upconversion experiments $[43,54]$. The finding from the target analysis that the X state serves as the energy-transfer donor to BChl implicates a significantly twisted structure in the mechanism of energy transfer.

\section{Intramolecular Charge Transfer and the $S^{*}$ State}

The torsional motions that a carotenoid undergoes on the $S_{2}$ potential surface after crossing the transition-state barrier are coupled to two additional degrees of freedom, from solvation and from distortion of the polyene out of the plane of conjugation, that would be expected to play important roles in the subsequent reaction dynamics. Both are directly associated with the formation of ICT character that occurs simultaneously with torsion.

Progress along the torsional coordinates from the barrier to the twisted minimum of the $S_{2}$ state potential energy surface is likely to be diffusive in character and strongly controlled by solvent friction. Twisting of a $\mathrm{C}=\mathrm{C}$ bond produces the electronic configuration of a biradical once the $\pi$ overlap of the two $2 p$ orbitals is lost. Because the two carbon atoms are inequivalent, however, there is a nonzero driving force for the transfer of one of the $2 p$ electrons across the bond from one carbon to another. The result is formally a charge-separated state, with the negatively charged length of the polyene favoring out-of-plane distortions towards a 
pyramidal conformation [55]. In simulations of cis-trans photochemistry in model PSBs, Hynes and coworkers[51] observed that the surrounding solvent's response to the increasing ICT character generally lags behind the torsional motions. An extension of this picture to carotenoids predicts that the lifetime of $S_{x}$ state in carotenoids will depend on the polar solvation timescale and the polarizability of the solvent.

In Figure 5, the development of a pyramidal conformation and a full ICT character is proposed to accompany the nonradiative transition from the $S_{2}$ state to the $S_{1}$ state. Calculations show that ionic states with doubly excited $\left(\pi^{*} \pi^{*}\right)$ configurations are found in polyenes near the twisted minimum adjacent to but at lower energy than the covalent states with singly excited $\left(\pi^{*}\right)$ configurations [21, $28,55,56]$. For example, the ionic states in ethylene [56] assume a pyramidal structure, with the out-of-plane distortion angle $\alpha=55^{\circ}$ and formally a lone pair electron configuration. Because of the sub-ps timescale associated with the conversion of the twisted $S_{x}$ structure, the crossing to the $S_{1}$ potential surface occurs far from thermal equilibrium. A narrowing and blue shifting of the ESA spectrum from the $S_{1}$ state on the $<1$ ps timescale is usually attributed to vibrational cooling on the $S_{1}$ state potential surface [57]; but note that de Weerd et al. [49] suggested that this process could be explained by torsional motions in reverse, towards a planar $\mathrm{S}_{1}$ state structure, which would have to be taken as being more stable than a twisted $\mathrm{S}_{1}$ conformation. Because of the ICT character that develops as a polyene twists, we suggest that this process is more likely to include diffusive relaxation of the conjugated backbone along the out-of-plane coordinates that lead to a pyramidal structure. In longer polyenes and carotenoids, the formation of an enhanced ICT character in the $\mathrm{S}_{1}$ state would be consistent with the presence of a bent or "kinked" [58] backbone that identifies a pyramidal distortion.

Carotenoids with carbonyl substitution such as fucoxanthin [59] and peridinin $[58,60-62]$ would be expected to be especially prone to the formation of an enhanced ICT character because the addition of an electron-withdrawing group in conjugation increases the tendency to fully transfer charge across a twisting $\mathrm{C}=\mathrm{C}$ bond. In peridinin, for example, Stark absorption experiments $[63,64]$ reveal that a very large change in permanent dipole moment accompanies the $S_{0} \rightarrow S_{2}$ optical transition, so even in the planar Franck-Condon structure the conjugated backbone has an asymmetric distribution of $\pi$ electrons along its length. The resulting enhancement of the asymmetry or polarization of the biradical character [55] would be expected to strongly favor a full transfer of charge and pyramidalization. The lifetime of the $S_{\mathrm{x}}$ state would be expected to be especially solvent dependent in carbonyl-substituted carotenoids because propagation along torsional coordinates would be especially slowed by solvent friction.

The final phases of nonradiative decay suggested in Figure 5 are associated with nonradiative decay from the $S_{1}$ state to the ground state, $S_{0}$. This step is formally a reverse charge-transfer reaction, the restoration of the biradical from the lonepair/ICT electronic configuration [51]. The driving force for this process would be expected to be very large because the product is the ground state, which lacks the $\pi^{*}$ 
character of the reactant. Because the reorganization energy for the reverse charge transfer would be comparable to that for the forward reaction, the increased driving force would very likely push the dynamics into the Marcus inverted regime [65], and accordingly the process would be expected to be very strongly retarded by polar solvation. Note that the $S_{1}$ state's pyramidal conformation would be retained in the $\mathrm{S}_{0}$ state structure initially formed. As is observed experimentally [66-68], the rate at which the $S_{1}$ state decays should be faster than the net recovery rate for the original, photoselected conformation in the ground state.

Figure 5 indicates that a spectroscopic distinction should be made between the pyramidal/ICT structure presumably present after vibrational cooling in the $\mathrm{S}_{1}$ state, the pyramidal biradical structure immediately produced by nonradiative decay from $S_{1}$ to $S_{0}$, and the formally planar $S_{0}$ structure that is eventually recovered after relaxation on the $S_{0}$ state potential surface. The photoinduced absorption spectrum from the pyramidal $S_{0}$ species would be expected to be blueshifted from the $S_{1}$ ESA spectrum owing to the $S_{1}-S_{0}$ energy gap near the $S_{1}$ pyramidal/ICT minimum energy structure, where a seam of the conical intersection with the $S_{0}$ potential surface would be found. But because the $S_{1}$ ESA spectrum and the photoinduced absorption spectrum from the $S_{0}$ state overlap spectrally and temporally, and because the latter shifts to the blue as its probability distribution diffuses down the ground state surface towards the photoselected ground state conformation, a number of timescales and spectra would be expected to be observed even in a given solvent [66-73]. The shortest of these, in the 2-5 ps regime, is likely to be associated with the blueshifting of the $S_{0}$ state distribution during the recovery back to the original photoselected conformation; the longest of these, in the $>10$ ps regime, would be associated with the nonradiative decay of $S_{1}$ to $\mathrm{S}_{0}$.

This scenario qualitatively accounts for some of what is known about the $S^{*}$ state, a dark intermediate state in carotenoids that has been the subject of controversy because many of the reported experimental observations are in apparent conflict. The reader is again referred to the review by Polívka and Sundström [4] for an introduction to this issue. The main point is that the $S^{*}$ state is usually identified as the origin of a shoulder on the short-wavelength side of the ESA spectrum from the $S_{1}$ state [19]. It has been assigned to the vibrationally excited $S_{0}$ state, as generated either by nonradiative decay from the $S_{1}$ state $[19,67]$ or by stimulated Raman scattering during resonant excitation of the $S_{2}$ state [66, 73]. An alternative suggestion is that $S^{*}$ is actually a low energy form of the $S_{1}$ state with a twisted conformation [69-71]. This assignment accounts for the finding that $S^{*}$ can serve as an energy donor to the BChl $\mathrm{Q}_{\mathrm{y}}$ state in the purple bacterial LH1 and LH2 complexes [72, 74]. In Figure 5, $S^{*}$ is assigned to the lowest energy form of the $S_{1}$ state, the pyramidal/ICT structure present after vibrational cooling. The finding that the $S^{*}$ ESA signal is much more intense in the LH2 complex than for carotenoids of the same conjugation length in solution [66] suggests the possibility that the twisted conformation favored by the binding site for the rhodopin glucoside in LH2 enhances the formation of $S^{*}$ [4]. Further, because the yield of intersystem crossing to the $\mathrm{T}_{1}$ state is apparently dramatically enhanced for carotenoids bound to 
proteins compared to solution, the nature of the $S^{*}$ conformation reached after vibrational cooling in the $S_{1}$ state and the dynamics that are possible are distinct in the two environments in an important way that affects the interaction between the $\mathrm{S}_{1}$ and $\mathrm{T}_{1}$ potential surfaces [75].

In closing this section, it should be made clear to the reader that many details of the involvement of conformational motions in the dynamics that accompany the $S_{2}$ to $S_{1}$ and $S_{1}$ to $S_{0}$ nonradiative decay processes remain to be determined experimentally. Figure 5 is intended to suggest that the most stable conformations in the $\pi^{*}$ excited states are twisted and pyramidal; formally these conformations correspond to biradical and pyramidal ICT structures, respectively. The simplest hypothesis, as depicted in the figure, is that a carotenoid proceeds after photoexcitation first along bond-length alternation coordinates and then along torsional coordinates to obtain a more stable excited-state conformation, so the reaction coordinate shown in the figure is principally intended to reflect progress along those two coordinates. As twisting occurs, however, the ICT character increases and the tendency to assume a pyramidal conformation should increase as well. Although the doorway to dynamics on the $>100$ fs timescale would be expected the ensemble of conformations reached near the twisted minimum of the $S_{2}$ surface, the subsequent dynamics amount to a multidimensional search of the $S_{1}$ potential surface along all of the intramolecular coordinates mentioned above, and each of these coordinates will be subject to friction from the solvent. The precise character of the excited state trajectory for a given system, then, requires specification of additional information, including the barrier height at the transition state between the planar and twisted conformations, which is determined principally by the length of the conjugated polyene and the ground-state conformation, and the solvent conditions, which control the coupling between torsions and charge transfer character. In agreement with a similar discussion in the de Weerd et al. [49] work about the character of twisting dynamics in the $S_{2}$ state, it would be an oversimplification to suggest that the $S_{2}$ and $S_{1}$ minima are purely twisted and pyramidal, and it is obviously possible that one part of the polyene is more twisted and another section bent (pyramidal), especially in a protein's binding site.

\section{Conclusions and Prospects}

An obvious general implication of the scheme outlined in Figure 5 is that the equilibrium distribution of ground state conformations observed in carotenoids in solution would be expected to have a significant impact on the nature of the excited state dynamics. In the simplest picture, by virtue of the Franck-Condon factors, the structure that is photoselected from the ground state ensemble controls the position and the potential energy of the Franck-Condon structure on the $S_{2}$ state surface. This choice will control the yield of the excited states that have enough energy to cross the transition-state barrier between planar and twisted structures. One would predict that in cases where the ground state absorption spectrum is heterogeneous owing to the presence of a distribution of conformers $[69,76]$, the tuning of the 
excitation laser in femtosecond spectroscopic experiments will have a significant effect on the observed response.

Rather than following the scheme shown in Figure 5, which applies to structures in solution that undergo excited state conformational motions, the bent and twisted carotenoid structures present in photosynthetic light-harvesting proteins are held in binding sites that favor prompt assumption of functional conformations with enhanced rates of energy transfer to $\mathrm{Chl}$ acceptors. In addition to exploiting control over the electrostatic environment of the carotenoid to tune the minimum-energy path it takes upon excitation on the $\mathrm{S}_{2}$-state potential energy surface, the protein can select conformations that are pretwisted enough to place the Franck-Condon structure past the transition-state barrier. One example of this is the situation in LH2 noted earlier that favors a stronger $\mathrm{S}^{*}$ signal than observed for carotenoids in solution [66]; the rhodopin glucoside chromophore is significantly twisted in the ground state [77]. As an additional example, the binding sites for peridinin in the peridinin-Chl $a$ protein appear to have pre-kinked structures (Figure 7) that are likely to enhance the ICT character by steering the relaxation of the $S_{2}$ state directly to pyramidal conformations. 


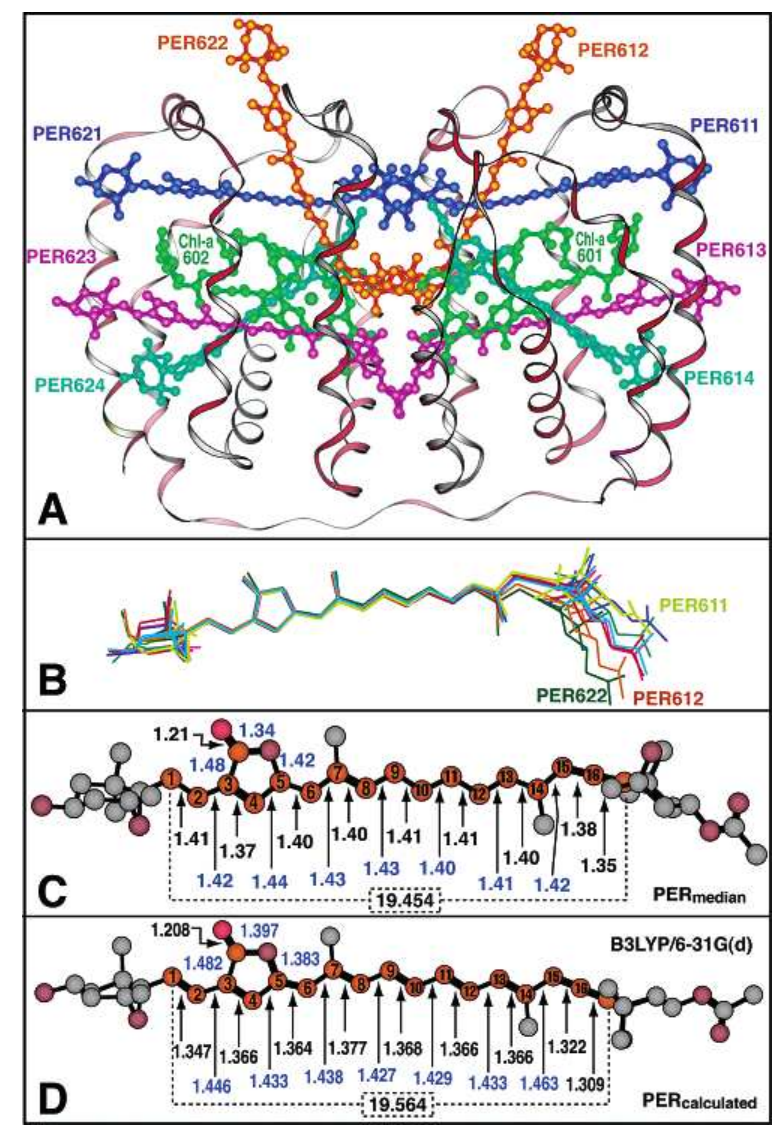

Figure 7. Structures of peridinin chromophores in the peridinin-Chl $a$ protein (PCP) from Amphidinium carterae: a.) Structures of the pigments and protein in one third of the PCP trimeric minimal unit, as rendered from PDB entry 1PPR [78]. The colors for the peridinin indicate the 2-fold symmetry axis. b.) Superimposed structures of all of the peridinins, with the orientations chosen to maximize the orbital overlap in the conjugated polyene. c.) Median structure for the peridinins. d.) Lowest-energy structure determined by an electronic structure calculation with density functional methods (B3LYP/6-31G(d)). From reference [61], copyright ACS, used with permission.

A test of the ideas presented in this contribution can be made with third-order nonlinear spectroscopic techniques with optical heterodyne detection, which are particularly well suited to address several of the outstanding questions about the nature of the dark states we have discussed. As shown in the work of Fleming and coworkers [79-82], conformational motions can be detected via the different time profiles exhibited by the absorption and dispersion parts of the third-order signal in transient grating experiments. Further, the contributions from double-quantum coherences that are superimposed on the population-dependent signals exhibited by carotenoids during nonradiative decay can be discerned by exploiting the control over time orderings that the choice of detection wavevector allows in stimulated photon echo experiments [83]. The additional information on donor-acceptor 
energy transfer pathways that is available from 2DES spectroscopy [84-87], as exploited in the Ostroumov et al. [15, 52] work discussed earlier, should contribute to considerable progress over the next few years in determining the key structural and mechanistic issues in light-harvesting proteins that mediate carotenoid to Chl energy transfer.

\section{Acknowledgment}

This work was supported by the Photosynthetic Systems program of Chemical Sciences, Geosciences and Biosciences Division, Office of Basic Energy Sciences, Office of Science, U.S. Department of Energy under Award Number DE-SC0010847. Work in the laboratory of HAF is supported by grants from the National Science Foundation (MCB-1243565) and the University of Connecticut Research Foundation. We thank Professor Benjamin Levine (Michigan State University) for several helpful discussions.

\section{Literature Cited}

[1] H.A. Frank, G.W. Brudvig, Redox functions of carotenoids in photosynthesis, Biochemistry, 43 (2004) 8607-8615. DOI: 10.1021/bi0492096.

[2] T. Polívka, V. Sundström, Ultrafast dynamics of carotenoids excited statesfrom solution to natural and artificial systems., Chem. Rev., 104 (2004) 2021-2071. DOI: $10.1021 / \mathrm{cr} 020674 \mathrm{n}$.

[3] V. Sundström, Photosynthetic light harvesting, charge separation, and photoprotection: the primary steps, in: L.O. Björn (Ed.) Photobiology: The Science of Life and Light, Springer, New York, 2007, pp. 289-319.

[4] T. Polívka, V. Sundström, Dark excited states of carotenoids: consensus and controversy, Chem. Phys. Lett., 477 (2009) 1-11. DOI: 10.1016/j.cplett.2009.06.011.

[5] H.A. Frank, R.J. Cogdell, Carotenoids in photosynthesis, Photochem. Photobiol., 63 (1996) 257-264. DOI: 10.1111/j.1751-1097.1996.tb03022.x.

[6] A.P. Shreve, J.K. Trautman, H.A. Frank, T.G. Owens, J.B. van Beek, A.C. Albrecht, On subpicosecond excitation energy transfer in light harvesting complexes (LHC): the B800-850 LHC of Rhodobacter sphaeroides 2.4.1, J. Lumin., 53 (1992) 179-186. DOI: 10.1016/0022-2313(92)90133-T.

[7] B.P. Kreuger, G.D. Scholes, R. Jimenez, G.R. Fleming, Electronic excitation transfer from carotenoid to bacteriochlorophyll in the purple bacterium Rhodopseudomonas acidophila, J. Phys. Chem. B, 102 (1998) 2284-2292. DOI: 10.1021/jp973062t.

[8] A. Damjanović, T. Ritz, K. Schulten, Excitation transfer in the peridininchlorophyll-protein of Amphidinium carterae, Biophys. J., 79 (2000) 1695-1705. DOI: 10.1016/S0006-3495(00)76422-8. 
[9] R.R. Birge, Two photon spectroscopy of protein-bound chromophores, Acc. Chem. Res., 19 (1986) 138-146. DOI: 10.1021/ar00125a003.

[10] R.L. Christensen, The electronic states of carotenoids, in: H.A. Frank, A.J. Young, G. Britton, R.J. Cogdell (Eds.) The Photochemistry of Carotenoids, vol. 8, Kluwer Academic Publishers, Dordrecht, 1999, pp. 137-159.

[11] M.Z. Papiz, S.M. Prince, T. Howard, R.J. Cogdell, N.W. Isaacs, The structure and thermal motion of the B800-850 LH2 complex from Rps. acidophila at $2.0 \AA$ resolution and $100 \mathrm{~K}$ : New structural features and functionally relevant motions, J. Molec. Biol., 326 (2003) 1523-1538. DOI: 10.1016/S0022-2836(03)00024-X.

[12] T. Förster, Zwischenmolekulare energiewanderung und fluoreszenz, Annu. Physik, 2 (1948) 55-75.

[13] G.D. Scholes, G.R. Fleming, On the mechanism of light harvesting in photosynthetic purple bacteria: B800 to B850 energy transfer, J. Phys. Chem. B, 104 (2000) 1854-1868. DOI: 10.1021/jp993435l.

[14] G. Cerullo, D. Polli, G. Lanzani, S. De Silvestri, H. Hashimoto, R.J. Cogdell, Photosynthetic light harvesting by carotenoids: Detection of an intermediate excited state, Science, 298 (2002) 2395-2398. DOI: 10.1126/science.1074685.

[15] E.E. Ostroumov, R.M. Mulvaney, R.J. Cogdell, G.D. Scholes, Broadband 2D electronic spectroscopy reveals a carotenoid dark state in purple bacteria, Science, 340 (2013) 52-56. DOI: 10.1126/science.1230106.

[16] C.C. Gradinaru, J.T.M. Kennis, E. Papagiannakis, I.H.M. van Stokkum, R.J. Cogdell, G.R. Fleming, R.A. Niederman, R. van Grondelle, An unusual pathway of excitation energy deactivation in carotenoids: singlet-to-triplet conversion on an ultrafast timescale in a photosynthetic antenna, Proc. Natl. Acad. Sci. U.S.A., 98 (2001) 2364-2369. DOI: 10.1073/pnas.051501298.

[17] H.C. Longuet-Higgins, L. Salem, The alternation of bond lengths in long conjugated molecules, Proc. R. Soc. London A, 251 (1959) 172-185. DOI: 10.1098/rspa.1959.0100.

[18] G. Orlandi, F. Zerbetto, M.Z. Zgierski, Theoretical analysis of spectra of short polyenes, Chem. Rev., 91 (1991) 867-891. DOI: 10.1021/cr00005a012.

[19] P.O. Andersson, T. Gillbro, Photophysics and dynamics of the lowest excited singlet state in long substituted polyenes with implications to the very long-chain limit, J. Chem. Phys., 103 (1995) 2509-2519. DOI: 10.1063/1.469672.

[20] W. Fuß, Y. Haas, S. Zilberg, Twin states and conical intersections in linear polyenes, Chem. Phys., 259 (2000) 273-295. DOI: 10.1016/S0301-0104(00)002007.

[21] B.G. Levine, T.J. Martínez, Ab initio multiple spawning dynamics of excited butadiene: role of charge transfer, J. Phys. Chem. A, 113 (2009) 12815-12824. DOI: 10.1021/jp907111u 
[22] M. Olivucci, I.N. Ragazos, F. Bernardi, M.A. Robb, A conical intersection mechanism for the photochemistry of butadiene. A MC-SCF study, J. Am. Chem. Soc., 115 (1993) 3710-3721. DOI: 10.1021/ja00062a042.

[23] P. Celani, F. Bernardi, M. Olivucci, M.A. Robb, Excited-state reaction pathways for $\backslash$ emph\{s-cis\} buta-1,3-diene, J. Chem. Phys, 102 (1995) 5733-5742. DOI: $10.1063 / 1.469304$.

[24] M.O. Trulson, R.A. Mathies, Excited-state structure and dynamics of isoprene from absolute resonance Raman intensities, J. Phys. Chem., 94 (1990) 5741-5747. DOI: 10.1021/j100378a026.

[25] U. Åberg, E. Åkesson, J.-L. Alvarez, I. Fedchenia, V. Sundström, Femtosecond spectral evolution monitoring the bond-twisting event in barrierless isomerization in solution, Chem. Phys., 183 (1994) 269-288. DOI: 10.1016/0301-0104(94)000220 .

[26] A. Yartsev, J.L. Alvarez, U. Åberg, V. Sundström, Overdamped wavepacket motion along a barrierless potential energy surface in excited state isomerization, Chem. Phys. Lett., 243 (1995) 281-289. DOI: 10.1016/0009-2614(95)00858-2.

[27] A. Sanchez-Galvez, P. Hunt, M.A. Robb, M. Olivucci, T. Vreven, H.B. Schlegel, Ultrafast radiationless deactivation of organic dyes: evidence for a two-state twomode pathway in polymethine cyanines, J. Am. Chem. Soc., 122 (2000) 2911-2924. DOI: $10.1021 /$ ja993985x

[28] B.G. Levine, C. Ko, J. Quenneville, T.J. MartÍnez, Conical intersections and double excitations in time-dependent density functional theory, Mol. Phys., 104 (2006) 1039-1051. DOI: 10.1080/00268970500417762.

[29] B. Dietzek, A. Yartsev, A.N. Tarnovsky, Watching ultrafast barrierless excited-state isomerization of pseudocyanine in real time, J. Phys. Chem. B, 111 (2007) 4520-4526. DOI: 10.1021/jp066471a.

[30] Z. Wei, T. Nakamura, S. Takeuchi, T. Tahara, Tracking of the nuclear wavepacket motion in cyanine photoisomerization by ultrafast pump-dump-probe spectroscopy, J. Am. Chem. Soc., 133 (2011) 8205-8210. DOI: 10.1021/ja110716b.

[31] A. Weigel, M. Pfaffe, M. Sajadi, R. Mahrwald, R. Improta, V. Barone, D. Polli, G. Cerullo, N.P. Ernsting, F. Santoro, Barrierless photoisomerisation of the "simplest cyanine": joining computational and femtosecond optical spectroscopies to trace the full reaction path, Phys. Chem. Chem. Phys., 14 (2012) 13350-13364. DoI: 10.1039/c2cp41522d.

[32] D.H. Waldeck, Photoisomerization dynamics of stilbenes, Chem. Rev., 91 (1991) 415-436. DOI: 10.1021/cr00003a007.

[33] G. Orlandi, W. Siebrand, Model for the direct photo-isomerization of stilbene, Chem. Phys. Lett., 30 (1975) 353-354. DOI: 10.1016/0009-2614(75)800054. 
[34] G. Orlandi, P. Palmieri, G. Poggi, An ab initio study of the cis-trans photoisomerization of stilbene, J. Am. Chem. Soc., 101 (1979) 3492-3497. DOI: $10.1021 / \mathrm{ja} 00507 \mathrm{a} 012$.

[35] G. Hohlneicher, B. Dick, Experimental determination of the low-lying excited A states of trans-stilbene, J. Photochem., 27 (1984) 215-231. DOI: 10.1016/00472670(84)85039-X.

[36] M. Garavelli, P. Celani, F. Bernardi, M.A. Robb, M. Olivucci, The $\mathrm{C}_{5} \mathrm{H}_{6} \mathrm{NH}_{2}{ }^{+}$ protonated Shiff base: an $a b$ initio minimal model for retinal photoisomerization, J. Am. Chem. Soc., 119 (1997) 6891-6901. DOI: 10.1021/ja9610895.

[37] M. Garavelli, T. Vreven, P. Celani, F. Bernardi, M.A. Robb, M. Olivucci, Photoisomerization path for a realistic retinal chromophore model: the nonatetraeniminium cation, J. Am. Chem. Soc., 120 (1998) 1285-1288. DOI: 10.1021/ja972695i.

[38] M. Garavelli, F. Negri, M. Olivucci, Initial excited-state relaxation of the isolated 11-cis protonated Schiff base of retinal: evidence for in-plane motion from ab initio quantum chemical simulation of the resonance Raman spectrum, J. Am. Chem. Soc., 121 (1999) 1023-1029. DOI: 10.1021/ja981719y.

[39] M.A. Robb, M. Olivucci, Photochemical processes: potential energy surface topology and rationalization using VB arguments, J. Photochem. Photobiol. A, 144 (2001) 237-243. DOI: 10.1016/S1010-6030(01)00453-1.

[40] D.S. Ruiz, A. Cembran, M. Garavelli, M. Olivucci, W. Fuß, Structure of the conical intersections driving the cis-trans photoisomerization of conjugated molecules, Photochem. Photobiol., 76 (2002) 622-633. DOI: 10.1562/00318655(2002)0760622SOTCID2.0.CO2.

[41] H.A. Frank, R.Z.B. Desamero, V. Chynwat, R. Gebhard, I. van der Hoef, F.J. Jansen, J. Lugtenburg, D. Gosztola, M.R. Wasielewski, Spectroscopic properties of spheroidene analogs having different extents of $\pi$-electron conjugation, J. Phys. Chem. A, 101 (1997) 149-157. DOI: 10.1021/jp962373l.

[42] D. Polli, G. Cerullo, G. Lanzani, S. De Silvestri, K. Yanagi, H. Hashimoto, R. Cogdell, Conjugation length dependence of internal conversion in carotenoids: role of the intermediate state, Phys. Rev. Lett., 93 (2004) 163002. DOI:

10.1103/PhysRevLett.93.163002.

[43] D. Kosumi, K. Yanagi, R. Fujii, H. Hashimoto, M. Yoshizawa, Conjugation length dependence of relaxation kinetics in $\beta$-carotene homologs probed by femtosecond Kerr-gate fluorescence spectroscopy, Chem. Phys. Lett., 425 (2006) 6670. DOI: 10.1016/j.cplett.2006.05.023.

[44] F. Rohlfing, D.D.C. Bradley, Optical non-linearity in $\beta$-carotene: new insight from electroabsorption spectroscopy, Chem. Phys. Lett., 227 (1997) 406-416. DOI: 10.1016/S0009-2614(97)00959-7. 
[45] D. Kosumi, M. Komukai, H. Hashimoto, M. Yoshizawa, Ultrafast dynamics of all-trans- $\beta$-carotene explored by resonant and nonresonant photoexcitations, Phys. Rev. Lett., 95 (2005) 213601-213604. DOI: 10.1103/PhysRevLett.95.213601.

[46] D. Kosumi, M. Fujiwara, H. Hashimoto, M. Yoshizawa, Ultrafast nonlinear optical responses induced by multiphoton excitation in all-trans- $\beta$-carotene: nonresonant excitation to the optically allowed $S_{2}$ state, J. Phys. Soc. Japan, 78 (2009) 104715. DOI: 10.1143/jpsj.78.104715.

[47] A. Zumbusch, G.R. Holtom, X.S. Xie, Three-dimensional vibrational imaging by coherent anti-Stokes Raman scattering, Phys. Rev. Lett., 82 (1999) 4142-4145. DOI: 10.1103/PhysRevLett.82.4142.

[48] B. Li, W.S. Warren, M.C. Fischer, Phase-cycling coherent anti-Stokes Raman scattering using shaped femtosecond laser pulses, Opt. Expr., 18 (2010) 2582525832. DOI: $10.1364 /$ OE.18.025825.

[49] F.L. de Weerd, I.H.M. van Stokkum, R. van Grondelle, Subpicosecond dynamics in the excited state absorption of all-trans- $\beta$-Carotene.pdf, Chem. Phys. Lett., 354 (2002) 38-43. DOI: 10.1016/S0009-2614(02)00095-7.

[50] W. Wohlleben, T. Buckup, J.L. Herek, R.J. Cogdell, M. Motzkus, Multichannel carotenoid deactivation in photosynthetic light harvesting as identified by an evolutionary target analysis, Biophys. J., 85 (2003) 442-450.

[51] J.P. Malhado, R. Spezia, J.T. Hynes, Dynamical friction effects on the photoisomerization of a model protonated Schiff base in solution, J. Phys. Chem. A, 115 (2011) 3720-3735. DOI: 10.1021/jp106096m.

[52] E.E. Ostroumov, R.M. Mulvaney, J.M. Anna, R.J. Cogdell, G.D. Scholes, Energy transfer pathways in light-harvesting complexes of purple bacteria as revealed by global kinetic analysis of two-dimensional transient spectra, J. Phys. Chem. B, 117 (2013) 11349-11362. DOI: 10.1021/jp403028x.

[53] D.M. Jonas, Two-dimensional femtosecond spectroscopy, Annu. Rev. Phys. Chem., 54 (2003) 425. DOI: 10.1146/annurev.physchem.54.011002.103907.

[54] D. Kosumi, M. Fujiwara, R. Fujii, R.J. Cogdell, H. Hashimoto, M. Yoshizawa, The dependence of the ultrafast relaxation kinetics of the $S_{2}$ and $S_{1}$ states in $\beta$ carotene homologs and lycopene on conjugation length studied by femtosecond time-resolved absorption and Kerr-gate fluorescence spectroscopies, J. Chem. Phys., 130 (2009) 214506. DOI: 10.1063/1.3147008.

[55] M. Klessinger, J. Michl, Excited states and photochemistry of organic molecules, VCH Publishers, New York, 1995.

[56] B.G. Levine, T.J. Martínez, Isomerization through conical intersections, Annu. Rev. Phys. Chem., 58 (2007) 613-634. DOI: 10.1146/annurev.physchem.57.032905.104612. 
[57] D.M. Niedzwiedzki, D.J. Sandberg, H. Cong, M.N. Sandberg, G.N. Gibson, R.R. Birge, H.A. Frank, Ultrafast time-resolved absorption spectroscopy of geometric isomers of carotenoids, Chem. Phys., 357 (2009) 4-16. DOI:

10.1016/j.chemphys.2008.07.011.

[58] Y. Pang, G.A. Jones, M.A. Prantil, G.R. Fleming, Unusual relaxation pathway from the two-photon excited first singlet state of carotenoids, J. Am. Chem. Soc., 132 (2010) 2264-2273. DOI: 10.1021/ja908472y.

[59] D. Kosumi, T. Kajikawa, S. Okumura, M. Sugisaki, K. Sakaguchi, S. Katsumura, $\mathrm{H}$. Hashimoto, Elucidation and control of an intramolecular charge transfer property of fucoxanthin by a modification of its polyene chain length, J. Phys. Chem. Lett., 5 (2014) 792-797. DOI: 10.1021/jz5000287.

[60] H.A. Frank, J.A. Bautista, J. Josue, Z. Pendon, R.G. Hiller, F.P. Sharples, D. Gosztola, M.R. Wasielewski, Effect of the solvent environment on the spectroscopic properties and dynamics of the lowest excited states of carotenoids, J. Phys. Chem. B, 104 (2000) 4569-4577. DOI: 10.1021/jp000079u.

[61] S. Shima, R.P. Ilagan, N. Gillespie, B.J. Sommer, R.G. Hiller, F.P. Sharples, H.A. Frank, R.R. Birge, Two-photon and fluorescence spectroscopy and the effect of environment on the photochemical properties of peridinin in solution and in the peridinin-chlorophyll-protein from Amphidinium carterae, J. Phys. Chem. A, 107 (2003) 8052-8066. DOI: 10.1021/jp022648z.

[62] H.M. Vaswani, C.P. Hsu, M. Head-Gordon, G.R. Fleming, Quantum chemical evidence for an intramolecular charge-transfer state in the carotenoid peridinin of peridinin-chlorophyll-protein, J. Phys. Chem. B, 107 (2003) 7940-7946. DOI: 10.1021/jp030086t.

[63] L. Premvardhan, E. Papagiannakis, R.G. Hiller, R. van Grondelle, The chargetransfer character of the $S_{0} \rightarrow S_{2}$ transition in the carotenoid peridinin is revealed by Stark spectroscopy, J. Phys. Chem. B, 109 (2005) 15589-15597. DOI: $10.1021 /$ jp052027g.

[64] T. Kusumoto, T. Horibe, T. Kajikawa, S. Hasegawa, T. Iwashita, R.J. Cogdell, R.R. Birge, H.A. Frank, S. Katsumura, H. Hashimoto, Stark absorption spectroscopy of peridinin and allene-modified analogues, Chem. Phys., 373 (2010) 71-79. DOI: 10.1016/j.chemphys.2010.01.018.

[65] G.L. Closs, J.R. Miller, Intramolecular long-distance electron transfer in organic molecules, Science, 240 (1988) 440-447. DOI:

10.1126/science.240.4851.440.

[66] W. Wohlleben, T. Buckup, H. Hashimoto, R.J. Cogdell, J.L. Herek, M. Motzkus, Pump-deplete-probe spectroscopy and the puzzle of carotenoid dark states, J. Phys. Chem. B, 108 (2004) 3320-3325. DOI: 10.1021/jp036145k. 
[67] T. Buckup, S. J., W. Wohlleben, J.L. Herek, H. Hashimoto, R.R.B. Correia, M. Motzkus, Pump-probe and pump-deplete-probe spectroscopies on carotenoids with $\mathrm{N}=9-15$ conjugated bonds, J. Chem. Phys., 125 (2006) 194505. DOI: $10.1063 / 1.2388274$.

[68] N. Christensson, F. Milota, A. Nemeth, J. Sperling, H.F. Kauffmann, T. Pullerits, J. Hauer, Two-dimensional electronic spectroscopy of $\beta$-carotene, J. Phys. Chem. B, 113 (2009) 16409-16419. DOI: 10.1021/jp906604j.

[69] D.M. Niedzwiedzki, J.O. Sullivan, T. Polívka, R.R. Birge, H.A. Frank, Femtosecond time-resolved transient absorption spectroscopy of xanthophylls, J. Phys. Chem. B, 110 (2006) 22872-22885. DOI: 10.1021/jp0622738.

[70] D. Niedzwiedzki, J.F. Koscielecki, H. Cong, J.O. Sullivan, G.N. Gibson, R.R. Birge, H.A. Frank, Ultrafast dynamics and excited state spectra of open-chain carotenoids at room and low temperatures, J. Phys. Chem. B, 111 (2007) 5984-5998.

[71] H. Cong, D.M. Niedzwiedzki, G.N. Gibson, H.A. Frank, Ultrafast time-resolved spectroscopy of xanthophylls at low temperature, J. Phys. Chem. B, 112 (2008) 3558-3567. DOI: 10.1021/jp0763681.

[72] H. Cong, D.M. Niedzwiedzki, G.N. Gibson, A.M. LaFountain, R. Kelsh, A.T. Gardiner, R.J. Cogdell, H.A. Frank, Ultrafast time-resolved carotenoid-tobacteriochlorophyll energy transfer in LH2 complexes from photosynthetic bacteria, J. Phys. Chem. B, 112 (2008) 10689-10703. DOI: 10.1021/jp711946w.

[73] T.R. Calhoun, J.A. Davis, M.W. Graham, G.R. Fleming, The separation of overlapping transitions in $\beta$-carotene with broadband 2D electronic spectroscopy, Chem. Phys. Lett, 523 (2012) 1-5. DOI: 10.1016/j.cplett.2011.10.051.

[74] E. Papagiannakis, J.T.M. Kennis, I.H.M. van Stokkum, R.J. Cogdell, R. van Grondelle, An alternative carotenoid-to-bacteriochlorophyll energy transfer pathway in photosynthetic light harvesting, Proc. Natl. Acad. Sci. U.S.A., 99 (2002) 6017-6022. DOI: 10.1073/pnas.092626599.

[75] D. Kosumi, S. Maruta, T. Horibe, Y. Nagaoka, R. Fujii, M. Sugisaki, R.J. Cogdell, H. Hashimoto, Ultrafast excited state dynamics of spirilloxanthin in solution and bound to core antenna complexes: Identification of the $\mathrm{S}^{*}$ and $\mathrm{T}_{1}$ states, J. Chem. Phys., 137 (2012) 064505. DOI: 10.1063/1.4737129.

[76] V. Lukeš, N. Christensson, F. Milota, H.F. Kauffmann, J. Hauer, Electronic ground state conformers of $\beta$-carotene and their role in ultrafast spectroscopy, Chem. Phys. Lett., 506 (2011) 122-127. DOI: 10.1016/j.cplett.2011.02.060.

[77] P.M. Dolan, D. Miller, R.J. Cogdell, R.R. Birge, H.A. Frank, Linear dichroism and the transition dipole moment orientation of the carotenoid in the LH2 antenna complex in membranes of Rhodopseudomonas acidophila strain 10050, J. Phys. Chem. B, 105 (2001) 12134-12142. DOI: 10.1021/jp010271b. 
[78] E. Hofmann, P.M. Wrench, F.P. Sharples, R.G. Hiller, W. Welte, K. Diederichs, Structural basis of light harvesting by carotenoids: peridinin-chlorophyll-protein from Amphidinium carterae, Science, 272 (1996) 1788-1791. DOI: 10.1126/science.272.5269.1788.

[79] Q.-H. Xu, G.R. Fleming, Isomerization dynamics of 1,1'-diethyl-4,4'-cyanine (1144C) studied by different third-order nonlinear spectroscopic measurements, J. Phys. Chem. A, 105 (2001) 10187. DOI: 10.1021/jp011924r.

[80] Q.-H. Xu, Y.-Z. Ma, G.R. Fleming, Heterodyne detected transient grating spectroscopy in resonant and non-resonant systems using a simplified diffractive optics method, Chem. Phys. Lett., 338 (2001) 254. DOI: 10.1016/S00092614(01)00281-0.

[81] Q.-H. Xu, Y.-Z. Ma, G.R. Fleming, Different real and imaginary components of the resonant third-order polarization revealed by optical heterodyne detected transient grating spectroscopic studies of crystal violet: model and experiment, J. Phys. Chem. A, 106 (2002) 10755. DOI: 10.1021/jp014714n.

[82] Q.-H. Xu, Y.-Z. Ma, I.V. Stiopkin, G.R. Fleming, Wavelength-dependent resonant homodyne and heterodyne transient grating spectroscopy with a diffractive optics method: solvent effect on the third-order signal, J. Chem. Phys., 116 (2002) 9333-9340. DOI: 10.1063/1.1473653.

[83] J. Kim, S. Mukamel, G.D. Scholes, Two-dimensional electronic doublequantum coherence spectroscopy, Acc. Chem. Res., 42 (2009) 1375-1384. DOI: 10.1021/ar9000795

[84] G.R. Fleming, G.D. Scholes, Physical chemistry - Quantum mechanics for plants, Nature, 431 (2004) 256-257.

[85] T. Brixner, J. Stenger, H.M. Vaswani, M. Cho, R.E. Blankenship, G.R. Fleming, Two-dimensional spectroscopy of electronic couplings in photosynthesis, Nature, 434 (2005) 625-628.

[86] E.L. Read, G.S. Engel, T.R. Calhoun, T. Mančal, T.K. Ahn, R.E. Blankenship, G.R. Fleming, Cross-peak-specific two-dimensional electronic spectroscopy, Proc. Natl. Acad. Sci. U.S.A., 104 (2007) 14203. DOI: 10.1073/pnas.0701201104.

[87] E. Collini, C.Y. Wong, K.E. Wilk, P.M.G. Curmi, P. Brumer, G.D. Scholes, Coherently wired light-harvesting in photosynthetic marine algae at ambient temperature, Nature, 463 (2010) 644. DOI: 10.1038/nature08811. 